\title{
Screen Arrangements and Interaction Areas for Large Display Work Places
}

\author{
Lars Lischke $^{1}$, Sven Mayer ${ }^{1}$, Katrin Wolf ${ }^{2}$, Niels Henze ${ }^{1}$, Harald Reiterer ${ }^{3}$, Albrecht Schmidt ${ }^{1}$ \\ ${ }^{1}$ University of Stuttgart (Pfaffenwaldring 5a, 70569 Stuttgart, Germany) \\ ${ }^{2}$ BTK University of Art and Design (Bernburger Str. 2425, 10963 Berlin) \\ ${ }^{3}$ University of Konstanz (Universittsstr. 10, 78457 Konstanz) \\ firstname.lastname@vis.uni-stuttgart.de ${ }^{1}$ - katrin.wolf@acm.org ${ }^{2}$ - harald.reiterer@ uni-konstanz.de 3
}

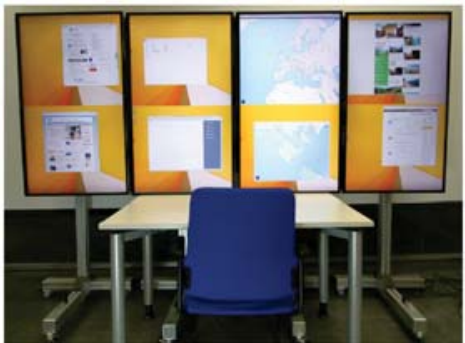

(a) Flat screen configuration

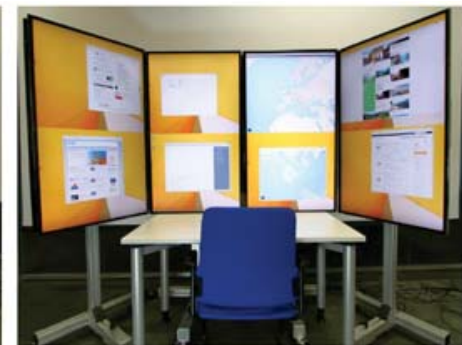

(b) Bow shaped screen configuration

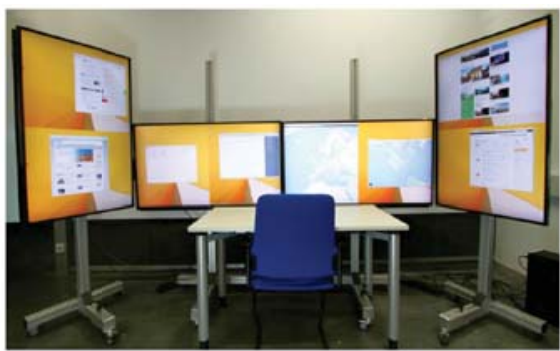

(c) Two plus two arrangement

Figure 1. Three screen configurations and applications we derived from a design study.

\begin{abstract}
Size and resolution of computer screens are constantly increasing. Individual screens can easily be combined to wallsized displays. This enables computer displays that are folded, straight, bow shaped or even spread. As possibilities for arranging the screens are manifold, it is unclear what arrangements are appropriate. Moreover, it is unclear how content and applications should be arranged on such large displays. To determine guidelines for the arrangement of multiple screens and for content and application layouts, we conducted a design study. In the study, we asked 16 participants to arrange a large screen setup as well as to create layouts of multiple common application windows. Based on the results we provide a classification for screen arrangements and interaction areas. We identified, that screen space should be divided into a central area for interactive applications and peripheral areas, mainly for displaying additional content.
\end{abstract}

\section{Author Keywords}

Large high-resolution displays; wall-sized display; user centred design study

\section{INTRODUCTION AND BACKGROUND}

Over the last two decades, the size and resolution of computer screens steadily increased. Today, display space can be arranged according to personal needs and preferences using out-of-the-box hardware. Graphic cards with multiple outputs and screens with a resolution of $3840 \times 2160$ px $(2160 p)$ or even higher are available on the consumer market. Most professional users of desktop computers already use two or even three screens connected to a single computer to increase their screen space. Using multiple screens enables to arrange them to user-defined physical display configuration. Already in 2001, Grudin [7] argued for using multiple screens to interact with complex applications. Previous research indicates that large displays can support the understanding of large data sets as large displays positively affect the users' productivity and satisfaction. Motivated by this, Hutchings and Stasko [8] interviewed 20 persons at their workplaces about using screen space. Based on these interviews, the authors proposed a classification how display space is used. The authors identified three categories how user manage their windows: (1) "maximizers", (2) "near maximizers" and (3) "careful coordinators". However, it is questionable if these results can transformed to large display space. In particular, the results provoke the question, how users would arranging content instead of maximizing windows.

In recent years, multiple research projects identified positive effects of large displays. Ball et al. [3] showed that users perform map search tasks faster on large displays and also when moving their body in front of the display compared to virtually moving the content through scrolling. Bi and Balakrishnan [5] conducted a long-term study to compare daily work 
on a single or dual screen setup with working on a large highresolution display (LHRD) with a size of $4.8 \times 1.8 \mathrm{~m}$ and a resolution of $6144 \times 2034 \mathrm{px}$. Their results revealed that users are faster while performing complex tasks including multiple windows on large displays. Also sensemaking can be supported by large display. Andrews et al. [1] observed in a lab study, using $4 \times 2$ grid of 30 inches LCDs, users typically spread out documents on large screens. This allows physical navigation and reduces mental load, because information can be compared without switching between multiple windows. Endert et al. [6] analyzed how LHRDs can be used for daily work. The authors compared different large-display setups for regular office work. They conclude that setup changes can have a large effect on users' performance.

Research focusing on form factors of displays for large display is mostly concerned about bezels between single screens. On one hand bezels might have a negative influence when users have to judge sizes of visual objects [16]. On the other hand thin bezels can support visual search [15]. Koppel et al. [14] show that observers' behaviour is strongly influenced by the form factor of public displays. To analyse this influence they used several screens mounted separately. With the trend of increasing display space also in office environments, understanding form factors for large displays in such environments is important.

Most previous work focuses on describing advantages of large displays, using a fully functional prototype. Often a standard graphical user interfaces (GUI) designed for single or dual screen setups, like Microsoft Windows is, used for analyzing large screen setups (e.g. $[1,5])$. We see a strong need to apply the findings of previous work together with the feedback from potential users to design tailor-made GUIs for large displays. Large displays allow fundamentally new arrangement of content. Therefore, we need detailed understanding of how users would use large interaction areas on wall-sized displays for daily work, independent from existing desktop solutions.

To understand how potential users would like to interact with content on a LHRD, Knudsen et al. [10] invited groups of experts from different domains. All invited experts work with large data sets on daily bases. This study was conducted in order to explore how these experts would like to use LHRDs. The authors used a whiteboard to prototype a large display and asked participants to draw their data and how they would interact with it. As a result, they identified the central area is used for generating ideas. Our work is inspired by these methods and we combined it with a common approach of requirements elicitation in interactive systems. In particular, using a low-tech approach is powerful as participants feel in full control over the setup. They are not constrained in their creativity and are empowered to make fundamental changes. However, Knudsen et al. [10] focused on interacting with one given shape of a large display in groups. In contrast, our work shows how single users would arrange wall-sized displays for their office work. Furthermore, we show if and how users would rearrange the display for a collaboration task and how they would use and interact the display space. In line with the work of Endert et al. [6] and Knudsen et al. [10] we see a need for new design guidelines for interacting wall-sized displays. Therefore, we conducted a user centered design study to understand how office workers, especially data analysts and programmer, would arrange large high-resolution screens to support them during their daily work.

\section{USER-CENTERED DESIGN STUDY}

The availability of LHRDs increases the digital interaction space for daily purposes dramatically. Hence, we seek understanding about how users want to use large display space. In addition, multi screen setups allow for combining and varying the form factors. For instance, freely arranging four screens provides a large number of different combinations, including form factors that are not available as a single screen yet. The screen layout and form factor possibilities that occur encourage us to envision a future where wall-sized displays in multiple shapes will be available.

We conducted a user-centered design study to gain a better understand how users would preferably arrange multiple large desktop screens and to gain knowledge about what layout would be best for application windows of various kinds. We used a repeated measures design presenting three different scenarios to all participants. We asked the participants to arrange screens as well as the application windows for the following three scenarios: (1) a software developing scenario ("Assume you are developing an android app"), (2) a text processing scenario ("Assume you are writing a longer report or thesis."), and (3) a visual collaboration data analysis scenario ("Assume you want to analyze and discuss a complex data set with your colleagues."). Consequently, we asked the participants for each scenario to perform two tasks. The first task was to arrange four screens. The second task was to place commonly used as well as the scenarios dependent application windows on the displays.

The user study was conducted in a room were we provide the participants with a regular office desk, a chair, and four lightweight cardboards. Each cardboard has the size of a screen with $50^{\prime \prime}$ diagonal $(1131 \mathrm{~mm} \times 697 \mathrm{~mm})$. Using four $50^{\prime \prime}$ screens has the advantage that the user can control the full display space without body movement [11]. Thus, we asked participants to create paper prototypes. Paper prototyping is a well-established technique for designing user interfaces [13]. We designed the study in line with the recommendations for prototyping multiple display environments by Bailey et al. [2]. We used four lightweight cardboards that participants could freely arrange in the study room. We used lightweight cardboard to not restrict participants to positions where today's heavy $50^{\prime \prime}$ screens would have been easy to place. Consequently, participants could design their own arrangement with low physical effort. We provided tripods and tape, but the use of it was not mandatory. The researcher who conducted the study offered physical support to arrange the cardboard pieces at the desired position. Thereby, height and orientation as well as rotation could easily be changed.

To get insights about how participants would arrange different windows on their arranged displays, we prepared a number of application window printouts. These printouts contained 
basic application windows for daily office work, including browsers, calendars, and e-mail software. Additionally, we prepared scenario-specific printouts for the three scenarios. Each application window was available on paper in two different sizes to see how dominant the participants wish each application window to be. Furthermore, we encouraged all participants to sketch other applications if they missed them in our prepared application set. Therefore, we provide the participants with different colored pens, A4 paper, and scissors. In such cases, we asked participants to describe the respective functionality.

After welcoming the participants, we explained the first scenario. As soon as all we clarified all questions about the scenario, we asked the participants to arrange the screens using the four cardboards in one desired arrangement. Then we requested the participants to attach the printed application windows on the cardboards using pins. Afterwards we repeated this procedure with the two other scenarios.

During scenario (1), we asked participants to design a setup for a situation in which she or he is focusing on software development (see Figure 2). In addition to basic application windows, we provided task-specific window printouts of Eclipse, a virtual Android device, and a log window. During scenario (2), we asked participants to set up the cardboards and printed application windows for an office task focusing on text writing. For this purpose and in addition to the basic applications, we provided task-specific printouts of Microsoft Word, Textmaker, larger text documents printed on A4 paper, and PDF documents presented in the Adobe Reader. Scenario (3) was a collaboration task. Here, we asked the participants to design for a situation where he or she has to analyze large data sets in a group of three or four people. To support this scenario, we provided printouts of different data plots, a data set in from of an Excel sheet and an SPSS output document. Additionally, participants could sketch their own ideas directly on the cardboards.

During the whole design process, participants could freely move in front of the cardboard displays. We also encouraged them to sit down during the design procedure as well as after they had finished a design task to experience the design from multiple perspectives.

Every participant took approximately 10 minutes per scenario to design a favored screen arrangement and a desired window placement. After designing for each scenario, the attending research recorded the designed screen through taking photos. Furthermore, participants explained every design of the screen arrangement as well as the window placement in a semi-structured interview. We compensated the participants for their time and effort with 10 EUR.

We selected computer scientists and engineers as they are used to perform complex tasks involving multiple applications, windows, and screens at the same time. We recruited 16 participants (10 male) from our university campus and via the computer science mailing list. The average age of the participants was 21.25 years $(S D=2.36)$. All had a background

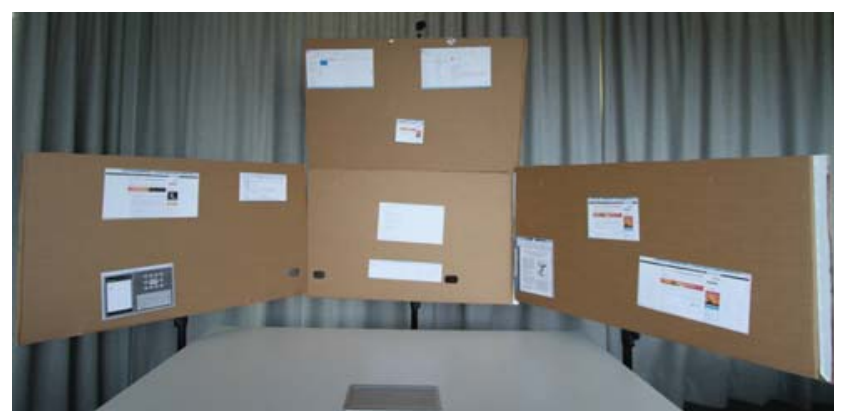

Figure 2. Cardboard and window arrangement designed by one of the participants during the study.

in engineering or computer science to ensure the participants were able to complete the first task.

\section{FINDINGS}

In this section, we present the findings from our design study in two steps. First, we focus on the arrangement of the individual screens. Second, we analyze the placement of the content displayed on the arranged LHRD.

\section{Screen arrangement}

In the first part of the study, we collected 19 screen arrangements from 16 participants. None of the participants changed the screen arrangement between scenario (1) and (2), the programming and text processing scenario. Three users changed the screen arrangement for scenario (3), analyzing and discussing a complex data set with colleagues. Using a bottomup analysis, we structured the 19 screen arrangements and grouped them by similarity, which results in the four screen arrangement categories: (a) screen band: all screens are placed directly next to each other in the same orientation. (b) screen block: all four displays are placed in a grid of $2 \times 2$ in landscape orientation. (c) cockpit arrangement: two displays in the center above each other in landscape orientation and one screen on each side of the stacked displays either in portrait or in landscape orientation. (d) - (f) two plus two arrangement: two screens in the main focus area have one orientation and the two screens in the periphery the other one. We present these six screen arrangements as sketches in Figure 4.

The screen band (a) was designed by 6 participants, the screen block (b) was proposed by 4 persons, the cockpit (c) by 5 , and 3 times the two plus two arrangement a screen band with an orientation change between focus and peripheral space was favoured. Two times arrangements were proposed only once. One of these arrangements was a variation of screens in landscape and portrait orientation. The other unique design was an asymmetrical arrangement containing two screens in landscape orientation behind the center of the desk and two screens in landscape orientation stacked above on the right side behind the desk. This arrangement can be seen as a hybrid between the screen band and the screen block.

The three participants who changed the screen arrangement for the collaborative scenario changed the setup to a screen 


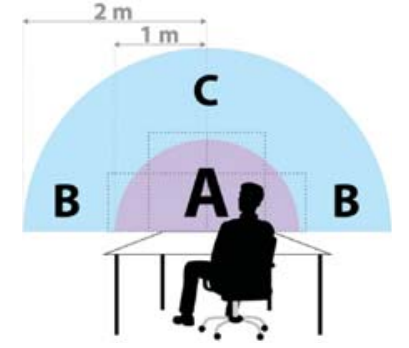

(a) Single user scenario

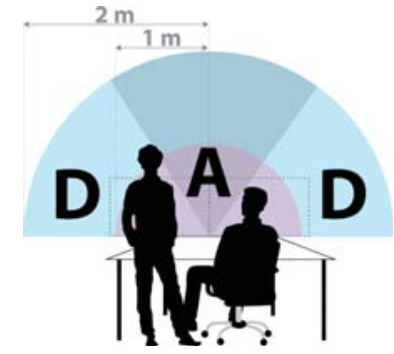

(b) Collaborative scenario
Figure 3. Interaction areas on LHRD. Single user scenario: (A) main focus, input $\&$ output (B) gathering information, output, reading $\&$ browsing (C) status information, communication. Collaborative scenario: (A) desktop input (D) creative working, discussing, sketching.

band or even isolated one screen to be able to stand with colleagues in front of that screen.

While six screen arrangements were designs as one display plane, the majority of the arrangements (13) were arranged in a bow shape. According to post-task comments, a reason for a bow shape was that the distance between the peripheral screens and the user is smaller compared to a planar setup. Moreover, three participants mentioned that they would also like to stand in front of their display from time to time. Two of them would like to sketch ideas and to take notes on the screens in the periphery. Therefore, they would like to have the peripheral screens equipped with technology that allows touch or digital pen input.

None of the participants saw a need for touch input on the whole display. This indicates the need of a screen arrangement, which enables users to work in two different body postures. All participants want to be able to interact with their digital environment in a sitting posture. Additionally, participants explained that they want to stand in front of the displays and work with absolute input e.g. touch input. Therefore, the participants would use the screens that are not placed directly behind the desk. These screens are accessible, because they are placed on the left or right side of the desk.

Surprisingly, no participant placed a screen laying on the desk, as an interactive tabletop. The reason could be that users place physical objects on their desk. These objects would cover the virtual work space. Another reason could be caused by the tripods. They could have motivated the participants to mount the screens instead of integrating them in the desk.

\section{Interaction Areas}

The majority of screen arrangements (15 of 19) combined all screens to one display unit to use it as a single display area, assuming the single display would not have any or only thin bezels. Only two participants separated a screen physically to be able to stand in front of the screen and discuss the displayed content with others. However, only two participants wanted to spread windows over the boundaries of screens. None of the participants wanted to fill the whole display with a single window. A few participants asked for displaying con- tent on one screen in full screen. Mostly, the participants used multiple windows on one screen. Participants stated that they would only use half of one screen for one window. This might have different reasons. On the one hand, a 50" cardboard could be perceivable as one unit. On the other hand, Grudin [7] sees a need to organize content on LHRDs. He argues for partitioning the screen space to keep an overview. In line with this, participants in our study stated that they would often like to compare the content of different windows. They would place two windows close to each other. The possibility to compare information from different sources without window switching is one of the main advantages the participants saw of LHRDs.

All window arrangements where independently categorized by two researchers. They conclude that the participants' arrangements of the windows indicates four areas that are used for different tasks (see Figure 3a). For the programming scenario (1) as well as for the text-processing scenario (2) all participants placed the mainly used windows in the middle of the display. Interestingly, all participants used the areas left hand side and right hand side of the display's center for web browsing or reading. All participants used multiple browser windows for gathering information. This led to the question, if they would still see a need for browser tabs. However, only one participant stated he would not use browser tabs anymore. All other would use tabs to group different topics, but they would use more browser windows and fewer tabs. The use of PDF viewers is comparable to the use of browser windows. All participants pined at least two windows of a PDF viewer, typically on the other end of the display, opposite to the web browser. Therefore, they used the browsers on the left of the center and PDF viewers on the right or the other way around.

Modern screens with 50" and 4K resolution allow displaying multiple A4 pages in original size. Even if the pages are magnified for a better readability multiple pages can comfortably be displayed. Thus, we asked participants how they would expect that a PDF viewer would display multiple pages at once. The majority (9) would expect to see three or four pages horizontally from left to right and then again three or four pages in a next row. All others would feel overwhelmed by seeing more than three pages at once. Therefore, one participant proposed to view the previous, the current and the next page at once. Thereby, the current page should be enlarged. In particular, for the text processing scenario some participants would like to use the areas left and right of the main focus area to stand in front of the displays and sketch ideas. Surprisingly, all participants had an exact idea, about applications, which they would never close. Half of the participants said that they would be distracted from their e-mail client or calendar. The other half would like to have as much status information as possible on screen. They would like to have multiple clients of their e-mail program and calendar on screen. However, all of them placed these windows above the main focus area. While sitting in front of the screen, the area above the main focus area is not in the field of vision. Nobody mentioned concerns about privacy issues when using such a large screen. Nevertheless, all participants hid their mail clients for the collaborative task. For this task, the participants would use the 
main focus area to edit the visualizations and to manipulate the data. They would use the areas left and right for the main focus area to display the visualizations (see Figure 3b). These results show interesting similarities to how smaller groups use space on interactive tabletops. Scott et al. [12] found three areas on interactive tabletops: (1) Personal space, in front of the user, where he or she is working independently form others. (2) Group space used for shared activity and the overall goal of the cooperation. (3) Storage space to place objects, which are currently not used.

\section{GUIDELINES}

As result of the presented study, we derive guidelines for designing LHRD workplaces and for designing user interfaces for such environments. The guidelines focus on a physical screen setup as well as on content arrangements.

\section{Symmetrical setups}

When designing LHRD workplaces, the user and desk should be placed in center of the setup. Users do not only prefer rectangular display setups. As shown in Figure 4, also screens in landscape and portrait mode are desired. Furthermore, all users prefer a symmetric arrangement of screens. If physical constrains allow for, users appreciate bow shaped setups. This has the advantage of equal viewing distances to all screens.

\section{Provide guidance}

Even if the study design clearly specified that the prototypes screens have no bezels, participants concerned about it. With a few exceptions in the center of the setup, participants did not spread single windows over multiple screens. We conclude that providing one large display without bezels is less important for users. In contrast, users make use of separated screens, to use them as "containers" to group related windows. This is in line with work presented by Grudin [7].

\section{Enable body movement}

It is beneficial to allow users to work in different body postures. This can help to get an overview over larger data sets [9] by physical navigation. Furthermore, when discussing display content users perceive standing in front of the display as more natural. Therefore, height adjustable display setups are beneficial to support single user as well as multi user workplaces. However, this require also more advanced input techniques, then mouse and keyboard. Direct touch input would allow multiple users to manipulate data at the same time. Due to this all touch, sensitive screens have to be in hand reaching distance for the users.

\section{User interfaces for large display environments}

Spreading content on LHRDs is intuitive and stacking becomes less important. For keeping overview new grouping techniques are needed. These techniques can be supported by functional areas on LHRDs.

\section{Support content spreading}

LHRD space allows users to spread content out, instead of stacking content. This has several implications. At first hiding content in tabs is not an appropriated content arrangement on large displays. Instead, content is spread over the whole display. However, this creates the need for new methods to connect multiple windows to one group. Each group should be displayed closely together and should provide functions applying to all windows, like close or hide all.

\section{Central focus}

When displays are larger than common desktop displays, users have more possibilities to arrange content spatially. This results in a central focus area. In this area is always in low middle of the setup. Users places applications, mostly interacted with, in this area. Such applications are used inter alia for text processing, programming or larger calculations. As these applications require most attention and interaction by the user, precise interaction techniques are needed in this area.

\section{Supportive content on the sides}

The display areas on the left and right of the focus area are used for supporting content. Users place web bowers or documents displaying documentations, manuals or additional existing data here. For shifting attention from the central focus area to the left or right side requires head or body movement. Furthermore, moving the pointing device to exterior areas is laborious. However, users do not need high precise input. A comparable concept for visualizations, named "focus plus context screens", provide high quality interaction in the focus and lower precise in the context area around [4]. User interfaces should assist users to place displayed content without overlaps and in a context-aware meaningful way.

In collaborative settings or for discussions there might be an attention shift in the direction of these areas. The focus area will be still used for preparation and adjustments. However, the results will be displayed in the supportive areas. In contrast to the concept of "focus plus context screens" [4] in this case high-resolution is also in these areas needed.

\section{Observing on the top}

Applications presenting notifications, messages and status information are placed by all users in the upper area of the display. These applications are mostly placed at the outside of the field of vision, when looking on the focus area. This has the advantage that notifications and messages do not distract users. Interface design should consider, how to avoid distractions, e.g. by fade not needed information out. However, it should be possible for important events to get user's attention. Changing color or blinking visual objects could realize this.

If a LHRD setup is designed for collaboration and discussions, privacy plays a more important role. Users appropriate mechanisms for easily hide private information, like calendars, emails or instant messages. One solution would be to extent applications with properties, which allow hiding or closing all of them with one action.

\section{IMPLEMENTATION OF A WORKING PROTOTYPE}

After analyzing results of the user study, we deployed a working prototype. We are focusing on constructing a wall-sized high-resolution display setup, which can be easily rearranged. To be able to test a wide range on setups, our system supports up to six screens. 


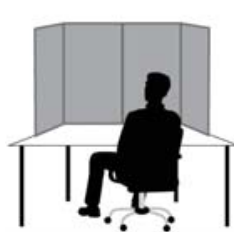

(a)

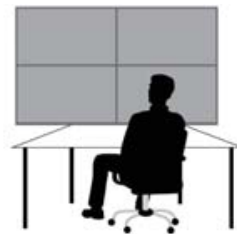

(b)

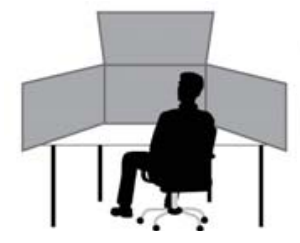

(c)

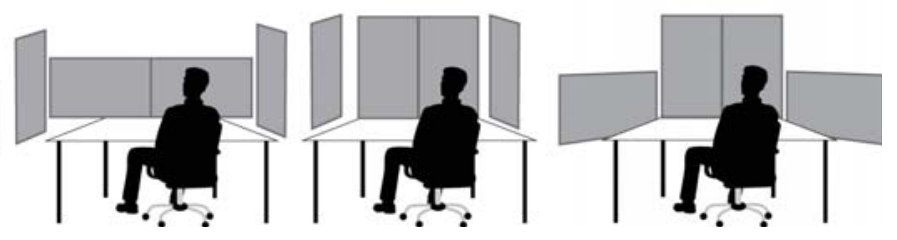

(d) (e)

Figure 4. Classifications of screen arrangements extracted from the design study: (a) screen band, (b) screen block, (c) cockpit arrangement, (d)-(f) two plus two arrangement.

We use up to six Panasonic TX-50AXW804 with 50" and 2160 p TV screens mounted on movable stands. This results in a display with a length of $4.04 \mathrm{~m}$, a high of $1.13 \mathrm{~m}$ and a total resolution of $12960 \times 3840 \mathrm{px}$. Besides resolution, refresh rate of the displayed image is important. Regularly desktop screens refresh the image at $60 \mathrm{~Hz}$. DisplayPort 1.2 and HDMI 2.0 are the only standards to drive a screen with 2160 p at $60 \mathrm{~Hz}$. Older DisplayPort or HDMI standards support only lower resolutions at $60 \mathrm{~Hz}$ refresh rate. In particular, TV screens do not always support 2160p at $60 \mathrm{~Hz}$. We use two AMD FirePro W9100 graphic cards in one workstation to drive up to six screens.

To ensure a high flexibility we mounted every screen to a custom movable aluminum stand, designed and constructed by feinarbyte. The stands are comparable to regular display stands, used at fairs and for presentations. In contrast to regular available stands, our stands are narrower, because regular stands hold screens in landscape orientation. To be able to arrange the screens in portrait orientation close to each other, the angle or the feeds of the stand is smaller. Every screen is mounted on a stand by a VESA $40 \times 40$ mount.

With the working prototypes (see Figure 1) we show that all screen arrangements shown in Figure 4 are feasible. In comparison between Figure 1a and $1 \mathrm{~b}$, the flat screen configuration, provides a better overview from a larger distance. However, even when sitting in a normal working distance, the viewing distance between the screens is varying. With a bow shaped configuration, the viewing distance is equal to all screens. Both setups require vertical head movement to view all content. This could cause neck tension. In contrast, the two plus two arrangement (see Figure 1c) uses landscape orientated screens for focused work. The lower height of the displays allows working without vertical head movement.

\section{CONCLUSION}

The contribution of this work is a detailed description of how users would work using large high-resolution displays in three common scenarios. We identified six screen arrangements for workplace setups, all favoring an arrangement in landscape format, even if some included portrait screen orientation. The findings suggest that users would like to work in different body postures with different input modalities. This calls for space to stand and sit in front of a large highresolution display. In addition, touch-sensitive input areas, where users can stand in front of the display, are needed. Furthermore, this work presents insights how users would arrange content on abundant display space. For single-use of the workspace, the participants employ the lower middle center for their main focus. Additional information will be displayed on the left and right side of the center area. Status information will be shown above the center area. In collaborative situations, areas are used comparably. Participants edit and prepare data in the center area of the screen and employ the space on the sides for presenting and discussing visualizations. To explain circumstances, users would like to be able to apply touch input on the regions left and right of the main input area.

Our contribution is a classification of screen arrangements and a definition of areas used for different activities performed on large high-resolution displays. In the next step, we will test different screen arrangements with a working prototype against each other to analyze which one is the most appealing for users working with complex data in single user and multi user scenarios. Furthermore, we will continue analyzing how content arrangement on wall-sized displays can be supported.

The working setup allows us to adjust and to test different screen arrangements with small effort. In the next step we will evaluate the six screen arrangements with a full functional system (see Figure 1). Based on this we will get more meaningful insights about different used display areas. Large high-resolution displays provide new possibilities office workspaces. Instead of sitting all the time, workers can also perform tasks while standing or moving. Reading or exploring data sets are predestinated tasks to be performed in different body postures. This can have a positive effect on motivation, task performance and worker's health. This calls for rethinking desktop metaphors fundamentally. On software side, instead of stacking, new widgets have to support content spreading and grouping. On hardware side, new controls have to allow implicit interaction (e.g. through changing body posture). Furthermore, large display setups have to support different input modalities for interacting with content on different display areas or in distinct body postures.

\section{Acknowledgement}

This work is partially funded by the European Community's H2020 Program under the funding scheme "FETPROACT1-2014: Global Systems Science (GSS)", grant agreement \# 641191 CIMPLEX. Furthermore, it was supported by the German Research Foundation (DFG) through projects C01, $\mathrm{C} 02$ and C04 of SFB/Transregio 161. This work is partly supported by DFG within SimTech Cluster of Excellence (EXC 310/2) at the University of Stuttgart. 


\section{REFERENCES}

1. Christopher Andrews, Alex Endert, and Chris North. 2010. Space to Think: Large High-resolution Displays for Sensemaking. In Proceedings of the SIGCHI Conference on Human Factors in Computing Systems (CHI '10). ACM, New York, NY, USA, 55-64. DOI : http://dx.doi.org/10.1145/1753326.1753336

2. Brian P. Bailey, Jacob T. Biehl, Damon J. Cook, and Heather E. Metcalf. 2008. Adapting paper prototyping for designing user interfaces for multiple display environments. Personal and Ubiquitous Computing 12, 3 (2008), 269-277. DOI :

http://dx.doi.org/10.1007/s00779-007-0147-2

3. Robert Ball, Chris North, and Doug A. Bowman. 2007. Move to Improve: Promoting Physical Navigation to Increase User Performance with Large Displays. In Proceedings of the SIGCHI Conference on Human Factors in Computing Systems (CHI '07). ACM, New York, NY, USA, 191-200. DOI : http://dx.doi.org/10.1145/1240624.1240656

4. Patrick Baudisch, Nathaniel Good, and Paul Stewart. 2001. Focus Plus Context Screens: Combining Display Technology with Visualization Techniques. In Proceedings of the 14th Annual ACM Symposium on User Interface Software and Technology (UIST '01). ACM, New York, NY, USA, 31-40. DOI : http://dx.doi.org/10.1145/502348.502354

5. Xiaojun Bi and Ravin Balakrishnan. 2009. Comparing Usage of a Large High-resolution Display to Single or Dual Desktop Displays for Daily Work. In Proceedings of the SIGCHI Conference on Human Factors in Computing Systems (CHI '09). ACM, New York, NY, USA, 1005-1014. DOI : http://dx.doi.org/10.1145/1518701.1518855

6. Alex Endert, Lauren Bradel, Jessica Zeitz, Christopher Andrews, and Chris North. 2012. Designing Large High-resolution Display Workspaces. In Proceedings of the International Working Conference on Advanced Visual Interfaces (AVI '12). ACM, New York, NY, USA, 58-65. DOI :

http://dx.doi.org/10.1145/2254556.2254570

7. Jonathan Grudin. 2001. Partitioning Digital Worlds: Focal and Peripheral Awareness in Multiple Monitor Use. In Proceedings of the SIGCHI Conference on Human Factors in Computing Systems (CHI 'O1). ACM, New York, NY, USA, 458-465. DOI : http://dx.doi.org/10.1145/365024.365312

8. Dugald Ralph Hutchings and John Stasko. 2004. Revisiting Display Space Management: Understanding Current Practice to Inform Next-generation Design. In Proceedings of Graphics Interface 2004 (GI '04). Canadian Human-Computer Communications Society, School of Computer Science, University of Waterloo, Waterloo, Ontario, Canada, 127-134. http: //dl.acm.org/citation. cfm?id=1006058.1006074
9. Mikkel R. Jakobsen and Kasper Hornbæk. 2015. Is Moving Improving?: Some Effects of Locomotion in Wall-Display Interaction. In Proceedings of the 33rd Annual ACM Conference on Human Factors in Computing Systems (CHI '15). ACM, New York, NY, USA, 4169-4178. DOI :

http://dx.doi.org/10.1145/2702123.2702312

10. Søren Knudsen, Mikkel Rønne Jakobsen, and Kasper Hornbæk. 2012. An Exploratory Study of How Abundant Display Space May Support Data Analysis. In Proceedings of the 7th Nordic Conference on Human-Computer Interaction: Making Sense Through Design (NordiCHI'12). ACM, New York, NY, USA, 558-567. DOI :

http://dx.doi.org/10.1145/2399016.2399102

11. Lars Lischke, Sven Mayer, Katrin Wolf, Niels Henze, Albrecht Schmidt, Svenja Leifert, and Harald Reiterer. 2015. Using Space: Effect of Display Size on Users' Search Performance. In Proceedings of the 33rd Annual ACM Conference Extended Abstracts on Human Factors in Computing Systems (CHI EA '15). ACM, New York, NY, USA, 1845-1850. DOI :

http://dx.doi.org/10.1145/2702613.2732845

12. Stacey D. Scott, M. Sheelagh T. Carpendale, and Kori M. Inkpen. 2004. Territoriality in Collaborative Tabletop Workspaces. In Proceedings of the 2004 ACM Conference on Computer Supported Cooperative Work (CSCW '04). ACM, New York, NY, USA, 294-303. DOI : http://dx.doi.org/10.1145/1031607.1031655

13. Carolyn Snyder. 2003. Paper prototyping: The fast and easy way to design and refine user interfaces. Morgan Kaufmann.

14. Maurice Ten Koppel, Gilles Bailly, Jörg Müller, and Robert Walter. 2012. Chained displays: Configurations of Public Displays can be used to Influence Actor-, Audience-, and Passer-By Behavior. In Proceedings of the 2012 ACM annual conference on Human Factors in Computing Systems - CHI '12. ACM Press, New York, New York, USA, 317. DOI :

http://dx.doi.org/10.1145/2207676.2207720

15. James R. Wallace, Daniel Vogel, and Edward Lank. 2014a. Effect of Bezel Presence and Width on Visual Search. In Proceedings of The International Symposium on Pervasive Displays (PerDis '14). ACM, New York, NY, USA, Article 118, 6 pages. DOI : http://dx.doi.org/10.1145/2611009.2611019

16. James R. Wallace, Daniel Vogel, and Edward Lank. 2014b. The Effect of Interior Bezel Presence and Width on Magnitude Judgement. In Proceedings of Graphics Interface 2014 (GI'14). Canadian Information Processing Society, Toronto, Ont., Canada, Canada, 175-182. http:

//dl. acm.org/citation. cfm?id=2619648.2619678 